Article

\title{
Modeling and Finite Element Analysis of Load-Carrying Performance of a Wind Turbine Considering the Influence of Assembly Factors
}

\author{
Jianmei Wang ${ }^{1, *}$, Ke Ning ${ }^{1}$, Liang Tang ${ }^{1}$, Reza Malekian ${ }^{2}$, Yinan Liang ${ }^{1}$ and Zhixiong $\mathrm{Li}^{3,4, *}$ \\ 1 School of Mechanical Engineering, Taiyuan University of Science and Technology, Taiyuan 030024, China; \\ ningke0811@163.com (K.N.); shinetang123@163.com (L.T.); benyinjushi@163.com (Y.L.) \\ 2 Department of Electrical, Electronic \& Computer Engineering, University of Pretoria, Pretoria 0002, \\ South Africa; reza.malekian@ieee.org \\ 3 School of Mechatronic Engineering, China University of Mining Technology, Xuzhou 221116, China \\ 4 School of Manufacturing \& Mechanical Engineering, UNSW, Sydney 2200, Australia \\ * Correspondence: wjmhdb@163.com (J.W.); zhixiongli@unsw.edu.au (Z.L.); Tel.: +86-1364-3459-006 (J.W.); \\ +61-0451-069-970 (Z.L.)
}

Academic Editor: Antonio Ficarella

Received: 3 February 2017; Accepted: 7 March 2017; Published: 18 March 2017

\begin{abstract}
In this work, a wind turbine shrink disk is used as the research object to investigate load-carrying performance of a multi-layer interference fit, and the theoretical model and finite element model are constructed. According to those models, a MW-level turbine shrink disk is designed, and a test device is developed to apply torque to this turbine shrink disk by hydraulic jack. Then, the circumferential slip between the contact surfaces is monitored and the slip of all contact surfaces is zero. This conclusion verifies the reasonability of the proposed models. The effect of the key influencing factors, such as machining deviation, assembly clearance and propel stroke, were analyzed. The contact pressure and load torque of the mating surfaces were obtained by building typical models with different parameters using finite element analysis (FEA). The results show that the minimum assembly clearance and the machining deviation within the machining range have little influence on load-carrying performance of multi-layer interference fit, while having a greater influence on the maximum assembly clearance and the propel stroke. The results also show that the load-carrying performance of a multiple-layer interference fit can be ensured only if the key factors are set within a reasonable design range. To avoid the abnormal operation of equipment caused by insufficient load torque, the propel stroke during practical assembly should be at least 0.95 times the designed propel stroke, which is significant in guiding the design and assembly of the multi-layer interference fit.
\end{abstract}

Keywords: wind turbine; shrink disk; multi-layer interference cylinder; assembly clearance; propel stroke

\section{Introduction}

An interference fit has structural advantages, such as simplicity, rigidity and effectiveness [1]. Additional fasteners can be omitted among the assemblies, thus preventing the weakening of joint strength [2]. An interference fit also has good aligning performance and large load-carrying capacity under complex operating conditions. The torque, axial force, or the combination of both can be transmitted. Such structures are mostly used in turbine shrink disks, cutting tool holders, wheels and bands for railway stock, and rotors for electric motors as well as for locating ball and roller bearings [3]. Many studies on the interference fit have been performed over the years. Manuel et al. [4] exploited finite element simulations, analytical approaches and experimental data in a study of fasteners with an 
interference fit. Boutoutaou et al. [5] illuminated the limitations of traditional theoretical calculation; they used finite element analysis (FEA) software for constructing defective and ideal models of mating surfaces, and comparative analysis showed that the defect on the mating surfaces had a great influence on the interference fit. Mack et al. [6] studied the influence of temperature on the interference fit structure, permanent plastic deformation and thermal expansion of surrounding pieces, concluding that a decrease in contact pressure would be beneficial. Jahed et al. [7] applied the simplex research method to obtain optimum distributions of thickness, stress and contact pressure for each layer of a multi-layer interference cylinder; as a result, the service life was prolonged. Sogalad [8] presented the influence of cylindricity and surface modification on load-carrying capacity of interference fitted assemblies. However, until now, there has been little research on the load-carrying performance of multi-layer interference fit, and the influencing factors for improving the load-carrying performance of the multi-layer interference cylinder have not yet been exploited.

To solve the above problem, this work gives the theoretical model and finite element model of the wind turbine shrink disk of a multi-layer interference fit. A test device has been developed to verify the reasonability of the proposed models. The effect of the key influencing factors, such as machining deviation, assembly clearance and propel stroke, were analyzed. The contact pressure of the mating surfaces and the load torque were obtained by building typical models with different parameters using FEA.

\section{Case Study}

As an important connection of a wind turbine spindle and a gearbox between the planetary frames, the shrink disk of a wind turbine aims to transfer large torque and withstand complex changeable loads. The whole transmission chain of a wind turbine will fail in the event of failure of the shrink disk. Here, the multi-layer interference mainly consists of a shaft, a sleeve, an inner ring and an outer ring. The half conical angle of the mating surface between the inner ring and the outer ring is $\beta=3^{\circ}$, as shown in Figure 1. The mating surface between the inner ring and the sleeve and that between the sleeve and the shaft are part of the clearance fit before assembly. The interference fit is formed through tightening the bolts, and the movement of the outer ring to inner ring along axial direction generates radial contact pressure. The continuing pressure of the inner ring shrinks the internal diameter of the sleeve; ultimately, the clearance of the mating surface between the shaft and the sleeve is eliminated and the interference fit is finally formed.

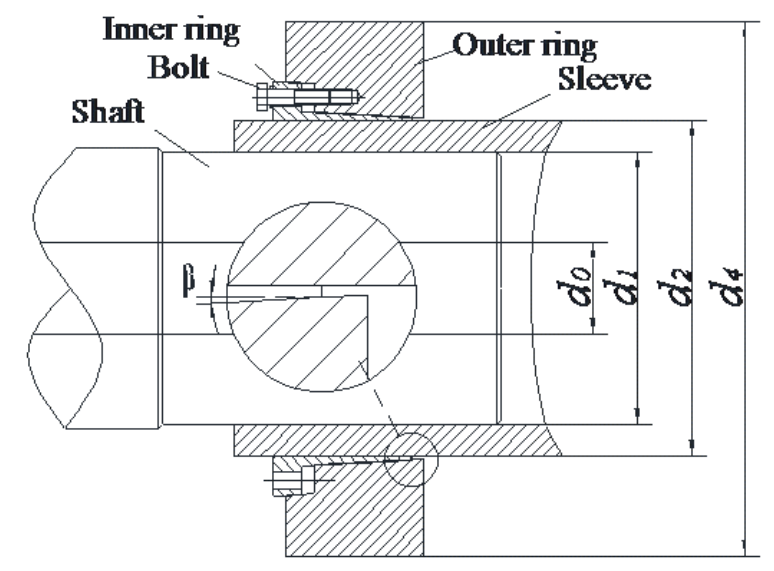

Figure 1. Schematic diagram of the turbine shrink disk.

When the turbine shrink disk is in operation, long conical surfaces between the inner ring and the outer ring account for the major interference fit. The interference of long conical surfaces has a decisive effect on the contact pressure of each mating surface, whereas short conical surfaces only form auxiliary connections. Thus, the results of long conical surfaces are mainly analyzed. The contact 
pressure and the interference magnitude of the inner ring and outer ring can be calculated by the contact pressure to transmit the rated torque $\mathrm{M}$ on the mating surface of shaft and sleeve, the required contact pressure to eliminate the assembly clearance between shaft and sleeve, and the required contact pressure between the sleeve and the inner ring.

The required contact pressure $P_{1}$ to transmit torque on the mating surfaces of the shaft and the sleeve is as follows [9]:

$$
P_{1}=\frac{2 M}{\mu_{1} \pi d_{1}^{2} l_{1}}
$$

where $\mu_{1}$ is the friction coefficient of the mating surfaces of the shaft and the sleeve; $d_{1}$ is the diameter of the mating surfaces of the shaft and the sleeve; and $l_{1}$ is the length of the contact surface of the shaft and the sleeve.

The required contact pressure $\Delta P_{1}$ to eliminate the assembly clearance between the shaft and sleeve is as follows [10]:

$$
\Delta P_{1}=\frac{R_{1} E_{2}\left(d_{2}^{2}-d_{1}^{2}\right)}{2 d_{1} d_{2}^{2}}
$$

where $R_{1}$ is the assembly clearance between the shaft and the sleeve; $E_{2}$ is the material elastic modulus of the sleeve; and $d_{2}$ is the diameter of the mating surfaces of the sleeve and the inner ring.

The required contact pressure $P_{2}$ on the external surface of sleeve is as follows:

$$
P_{2}=P_{1}+\Delta P_{1}
$$

When the assembly clearance on the mating surfaces of the sleeve and the inner ring is eliminated, the contact pressure $\Delta P_{2}$ is required, which is given as:

$$
\Delta P_{2}=\frac{R_{2} E_{3}\left(d_{3 l}^{2}-d_{2}^{2}\right)}{2 d_{2} d_{3 l}^{2}}
$$

where $R_{2}$ is the assembly clearance of the mating surfaces of the sleeve and the inner ring; $E_{3}$ is the material elastic modulus of the inner ring; $d_{3 l}$ is the average diameter of the mating surfaces of the inner ring and the outer ring.

The external surface of the inner ring exerts the contact pressure of $P_{3}$, which is given as:

$$
P_{3}=P_{2}+\Delta P_{2}
$$

The magnitude of interference $\delta_{3}$ on the mating surfaces of the inner ring and the outer ring is given as follows [9]:

$$
\delta_{3}=P_{3} d_{3}\left(\frac{C_{3}}{E_{3}}+\frac{C_{4}}{E_{4}}\right)
$$

where $C_{3}$ and $C_{4}$ are coefficients expressed as $C_{3}=\frac{d_{3}^{2}+d_{0}{ }^{2}}{d_{3 l}^{2}-d_{0}{ }^{2}}-v_{b}$ and $C_{4}=\frac{d_{4}^{2}+d_{3 l}{ }^{2}}{d_{4}^{2}-d_{3 l^{2}}}+v_{4} ; v_{b}$ is Poisson's ratio of the shaft, sleeve and inner ring; and $v_{4}$ is Poisson's ratio of the outer ring.

\section{Key Influencing Factors}

\subsection{Assembly Clearance}

The mating surface between the inner ring and the sleeve and that between the sleeve and the shaft form a clearance fit before assembly, and the elimination of clearance between mating surfaces forms the interference fit during assembly. This clearance can weaken the pressure transmission between mating surfaces and affects the performance of the interference fit [11,12]. Deviation is inevitable in practical manufacturing, with the actual clearance of the mating surfaces being between the minimum and the maximum clearance. According to the sizes of some types of turbine shrink 
disks, the minimum clearance between the sleeve and the shaft is chosen to be $0.022 \mathrm{~mm}$, and the maximum clearance is set as $0.136 \mathrm{~mm}$; the minimum clearance between the inner ring and sleeve is chosen to be $0.08 \mathrm{~mm}$, and the maximum clearance is set as $0.24 \mathrm{~mm}$.

The contact pressure $P_{3}$ between the inner ring and the outer ring can be deduced by Equations (2)-(5).

$$
\begin{gathered}
P_{3}=P_{2}+\left[\frac{E_{3}\left(d_{3 l}^{2}-d_{2}^{2}\right)}{2 d_{2} d_{3 l}^{2}}\right] R_{2} \\
P_{3}=P_{1}+\left[\frac{E_{2}\left(d_{2}^{2}-d_{1}^{2}\right)}{2 d_{1} d_{2}^{2}}\right] R_{1}+\left[\frac{E_{3}\left(d_{3 l}^{2}-d_{2}^{2}\right)}{2 d_{2} d_{3 l}^{2}}\right] R_{2}
\end{gathered}
$$

Using Equation (6), it can be deduced that contact pressure $P_{3}$ is a constant value if the magnitude of interference $\delta_{3}$ is certain. Using Equations (7) and (8), it can be deduced that contact pressure $P_{1}$ is inversely proportional to assembly clearances $R_{1}$ and $R_{2}$; contact pressure $P_{2}$ is inversely proportional to assembly clearance $R_{2}$, when contact pressure $P_{3}$ is a constant. Model 1, Model 2 and Model 3 , with the same magnitude of interference $\delta_{3}$ in Table 1, are used to compare the effect of the assembly clearance. Model 1 represents the case in which each mating surface is under the ideal condition, Model 2 corresponds to the case in which the clearance of each of the mating surfaces is at the minimum value, and Model 3 represents the case in which the clearance of mating surfaces is at the maximum value.

\begin{tabular}{|c|c|c|c|c|c|}
\hline \multirow[b]{2}{*}{$\begin{array}{l}\text { Model } \\
\text { Category }\end{array}$} & \multirow[b]{2}{*}{$\begin{array}{l}\text { Model } \\
\text { Number }\end{array}$} & \multicolumn{2}{|c|}{ Assembly Clearance (mm) } & \multirow{2}{*}{$\begin{array}{c}\text { Machining } \\
\text { Deviation }(\mathrm{mm}) \\
\text { Mating Surface of the } \\
\text { Inner Ring and the } \\
\text { Outer Ring }\end{array}$} & \multirow[b]{2}{*}{$\begin{array}{c}\text { Propel } \\
\text { Stroke }(\mathrm{mm})\end{array}$} \\
\hline & & $\begin{array}{l}\text { Mating Surface } \\
\text { of the Shaft and } \\
\text { the Sleeve }\end{array}$ & $\begin{array}{l}\text { Mating Surface of } \\
\text { the Sleeve and the } \\
\text { Inner Ring }\end{array}$ & & \\
\hline \multirow{3}{*}{$\begin{array}{l}\text { Assembly } \\
\text { clearance }\end{array}$} & $1 \#$ & & 0 & \multirow{3}{*}{$d_{A}, d_{B}, d_{C}, d_{D}$} & \\
\hline & $2 \#$ & 0.022 & 0.08 & & \\
\hline & $3 \#$ & 0.136 & 0.24 & & \\
\hline \multirow{2}{*}{$\begin{array}{l}\text { Machining } \\
\text { deviation }\end{array}$} & $4 \#$ & \multirow{2}{*}{\multicolumn{2}{|c|}{0}} & $\begin{array}{c}d_{A}-0.062, d_{B}-0.062 \\
d_{C}+0.062, d_{D}+0.062\end{array}$ & $\mathrm{BD}$ \\
\hline & $5 \#$ & & & $\begin{array}{c}d_{A}+0.062, d_{B}+0.062 \\
d_{C}-0.062, d_{D}-0.062\end{array}$ & \\
\hline \multirow{2}{*}{ Propel stroke } & $6 \#$ & \multirow{2}{*}{\multicolumn{2}{|c|}{0}} & \multirow{2}{*}{$d_{A}, d_{B}, d_{C}, d_{D}$} & $\mathrm{BD}_{1}$ \\
\hline & $7 \#$ & & & & $\mathrm{BD}_{2}$ \\
\hline
\end{tabular}

Table 1. Dimensions of mating surfaces.

\subsection{Machining Deviation}

The interference magnitude between the inner ring and the outer ring is the key parameter to the load-carrying capacity of the turbine shrink disk. There inevitably exists certain machining deviation during the processing of both rings, which causes deviation of the interference magnitude between the actual value and the design value. The larger interference magnitude can easily produce stress concentration, whereas the smaller values cannot ensure the reliability of the interference fit.

As shown in Figure 2, the influence of the machining deviation on the load-carrying capacity of the turbine shrink disk was analyzed through the dimensions of key points A, B, C, and D on the mating surface of the inner ring and the outer ring. The design diameter dimensions of each point are $d_{A}, d_{B}, d_{C}$ and $d_{D}$. According to the dimensions of the turbine shrink disk, the machining deviation of the inner ring and the outer ring along the diameter direction is given as $\pm 0.062 \mathrm{~mm}$. Moreover, Figure 2 shows the enlarged diagram of the relations between propel stroke, interference magnitude, and critical points $D_{1}$ and $D_{2}$ that are produced as the inner ring approaches the right ahead in turn. 


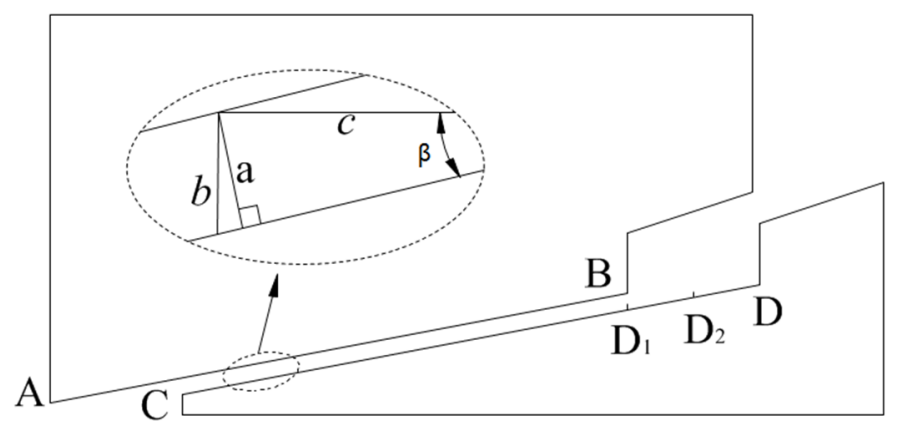

Figure 2. Schematic diagram of the mating surfaces between the inner ring and the outer ring.

The machining deviation will directly affect the interference magnitude. From Equation (7), we know that contact pressure $P_{3}$ is proportional to the interference magnitude. From Equations (7) and (8), we can deduce that contact pressures $P_{1}$ and $P_{2}$ are proportional to contact pressure $P_{3}$ when the assembly clearance is certain, and contact pressures $P_{1}$ and $P_{2}$ are proportional to the magnitude of interference $\delta_{3}$. Thus, the machining deviation will affect contact pressures $P_{1}$ and $P_{2}$. Model 1, Model 4 and Model 5, with the same assembly clearance stated in Table 1, are used to study the effect of machining deviation. Model 4 and Model 5 have the minimum and maximum interference magnitudes, respectively, which result from machining deviation on the mating surfaces of the inner ring and the outer ring.

\subsection{Propel Stroke}

The outer ring moves toward the inner ring in the axial direction during assembly, and the interference magnitude on the mating surface of the inner ring and outer ring directly relates to the distance of axial movement. If the distance of the axial movement of the outer ring does not meet the design value, then the interference magnitude on the mating surface of the inner ring and outer ring will be less than the design value, which will inevitably result in the turbine shrink disk being unable to transmit the rated torque. According to the enlarged diagram in Figure 2, the relationship between the propel stroke of the outer ring and the interference magnitude is as below:

$$
\begin{aligned}
& a=\frac{b}{\cos \beta} \\
& c=\frac{a}{\sin \beta}
\end{aligned}
$$

Using Equations (8) and (10),

$$
c=\frac{b}{\sin \beta \cos \beta}
$$

Setting $L$ as the propel stroke, $\beta$ as the half angle, $\delta_{3 L}$ as the magnitude of interference, there exists a relationship between the interference magnitudes and the propel stroke, as follows:

$$
L=\frac{\delta_{3 L}}{2 \sin \beta \cos \beta}=k_{L \delta_{3 L}} \delta_{3 L}
$$

where $k_{L \delta_{3 L}}$ is the scale factor of the propel stroke $L$ and interference magnitude $\delta_{3 L}$.

According to Equation (12), in addition to the machining deviation and assembling clearance of the inner ring and the outer ring, the effect on the propel stroke of the outer ring must be taken into account during the design. Different propel strokes based on ideal Model 1 are set in Table 1, as shown in Figure 2. When point $B$ of the outer ring approaches inner point $D$, it is called full propulsion, which sets $L$ as propel stroke $B D$. When point $B$ approaches $D_{1}$, the propel stroke $B_{1}$ is $0.8 L$. When point $B$ approaches $\mathrm{D}_{2}$, the propel stroke $\mathrm{BD}_{2}$ is $0.9 \mathrm{~L}$. 
From Equation (12), we can deduce that the interference magnitude $\delta_{3 L}$ and the propel stroke $L$ are directly proportional. From Equation (6), the contact pressure $P_{3}$ is proportional to interference magnitude $\delta_{3 L}$. From Equations (7) and (8), contact pressures $P_{1}$ and $P_{2}$ are proportional to contact pressure $P_{3}$. Thus, contact pressures $P_{1}$ and $P_{2}$ will increase with the increasing propel stroke $L$ and decrease with the decreasing propel stroke L. Model 1, Model 6 and Model 7 are used to compare the effect of the propel stroke. Model 6 has the least propel stroke $\mathrm{BD}_{1}$, and Model 7 has a lesser propel stroke $\mathrm{BD}_{2}$.

\section{Finite Element Model}

The general information regarding the finite element model based on Table 1 is as follows. Taking into account the geometric symmetry of the turbine shrink disk and the symmetry of the loads on various components, a two-dimensional axisymmetric model is established with FEA software ABAQUS. Four-node asymmetrically reduced integral unit CAX4R and the contact algorithm of penalty function method is adopted in the model $[13,14]$. The mesh size of shaft, sleeve, inner ring and outer ring is 2, 1, 2 and $2 \mathrm{~mm}$, respectively. The element shape is quad and the algorithm is medial axis algorithm. According to the actual working conditions, the right end of the inner ring is set with an axial constraint; the left end of the sleeve and the right end of the shaft are set with the fixed-end constraint [15]. The boundary constraints and mesh distribution of the finite element model of the turbine shrink disk are shown in Figure 3. There are three pairs of mating surfaces in this model. Each mating surface is defined as a limited slip. The mating surface of the inner ring and the outer ring is coated with molybdenum disulfide grease during assembly, and the friction coefficient is set to be 0.9 , whereas the friction coefficient of the other mating surfaces is set as 0.15 . The propel stroke of the inner ring $L$ is $22.88 \mathrm{~mm}$. The design interference magnitude on the mating surface of the inner ring and the outer ring is $2.474 \mathrm{~mm}$. The parameters of a type of turbine shrink disk are shown in Table 2.

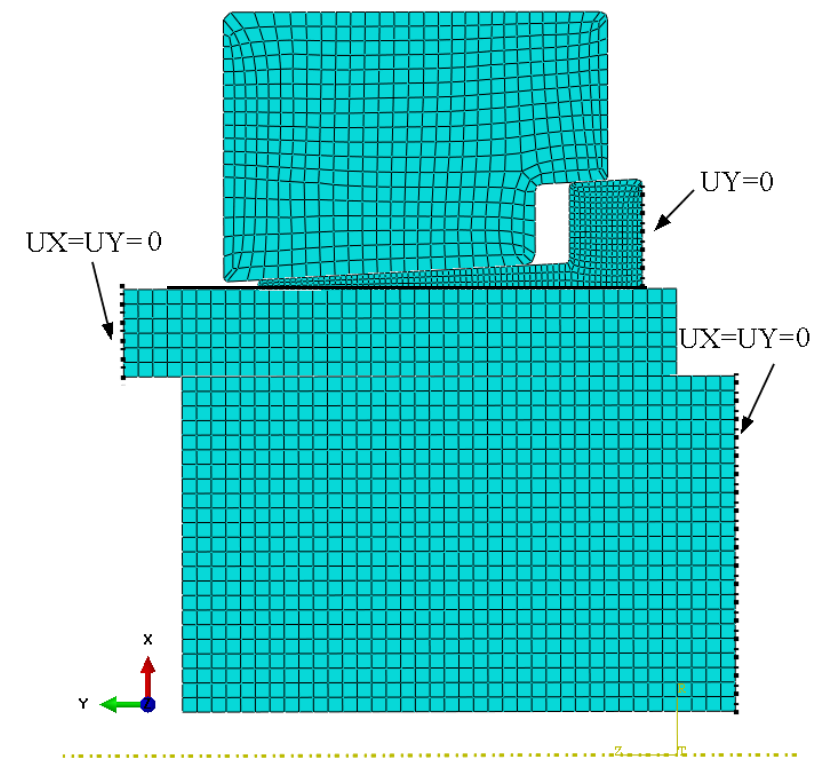

Figure 3. The boundary constraints and mesh distribution of the finite element model of the turbine shrink disk. 
Table 2. Parameters of wind turbine shrink disk.

\begin{tabular}{lc}
\hline \multicolumn{1}{c}{ Parameters } & Value \\
\hline Inside diameter of shaft, $d_{0}(\mathrm{~mm})$ & 60 \\
Diameter of the mating surfaces of shaft and sleeve, $d_{1}(\mathrm{~mm})$ & 520 \\
Diameter of the mating surfaces of sleeve and inner ring, $d_{2}(\mathrm{~mm})$ & 640 \\
Average diameter of long conical surfaces of inner ring, $d_{3}(\mathrm{~mm})$ & 663.715 \\
Outside diameter of outer ring, $d_{4}(\mathrm{~mm})$ & 1020 \\
Length of the contact surface of shaft and sleeve, $l_{1}(\mathrm{~mm})$ & 255 \\
Rated torque on mating surface of shaft and sleeve, $M(\mathrm{~N} \cdot \mathrm{m})$ & 2800 \\
Material elastic modulus of shaft, inner ring and outer ring $(\mathrm{GPa})$ & 210 \\
Material elastic modulus of sleeve $(\mathrm{GPa})$ & 180 \\
Poisson's ratio & 0.300 \\
\hline
\end{tabular}

\section{Test Verification}

Based on the design theory of this work, a MW-level turbine shrink disk was designed to verify the reasonability of the proposed theoretical model and finite element model, and the parameters are given in Table 2. A test device was developed for performance test, as shown in Figure 4. The test gradually applies torque to the turbine shrink disk by hydraulic jack and supporting bracket. In the performance test of the shrink disk, the holding pressure time is set as three minutes based on test experience. The circumferential slip between the contact surfaces is monitored using the method of fixed reference line, the slip of all contact surfaces is zero, which shows this design size can transfer the applied torque and can meet the requirements of the given working conditions; the specific data is given in Table 3. The rated torque of shaft transmission is $T_{0}=2800 \mathrm{kN} \cdot \mathrm{m}$, as shown in Table 2.

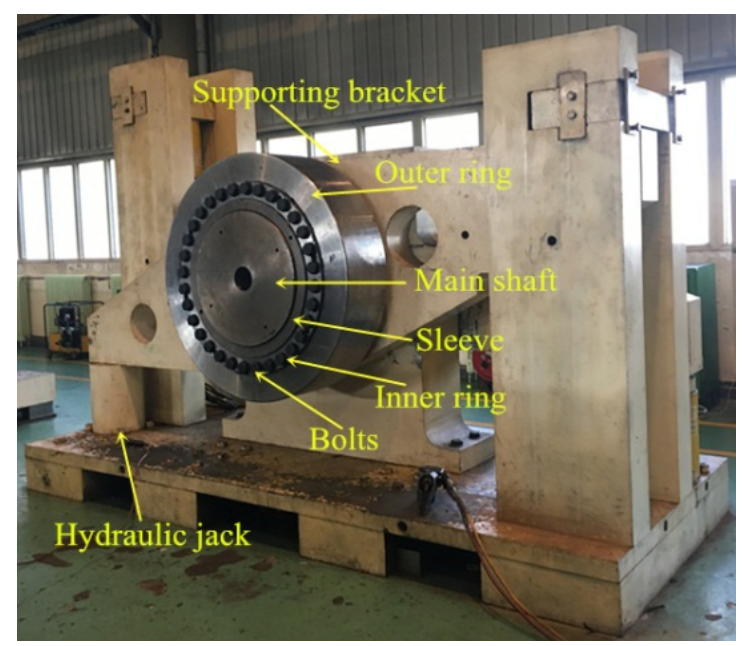

Figure 4. Test bench of turbine shrink disk.

Table 3. Static torque detection data.

\begin{tabular}{|c|c|c|}
\hline Torque Value $T(\mathrm{kN} \cdot \mathrm{m})$ & Circumferential Slip (S/mm) & Holding Pressure Time ( $t / \mathrm{min})$ \\
\hline $0.4 T_{0}$ & 0 & 3 \\
\hline $0.5 T_{0}$ & 0 & 3 \\
\hline $0.65 T_{0}$ & 0 & 3 \\
\hline $0.8 T_{0}$ & 0 & 3 \\
\hline$T_{0}$ & 0 & 3 \\
\hline $1.2 T_{0}$ & 0 & 3 \\
\hline
\end{tabular}




\section{Results and Discussions}

Through the above-described modeling method, models with different assembly clearance, machining deviation, and propel stroke were compared with the ideal model, and the influences of each factor on the contact pressure of the mating surfaces were analyzed. The contact pressure is generated between each of the mating surfaces during assembly, which directly affects the working performance of the mating surfaces [16]. Figure 5 gives the distribution of the Von Mises stress of the turbine shrink disk using the ideal model [17].

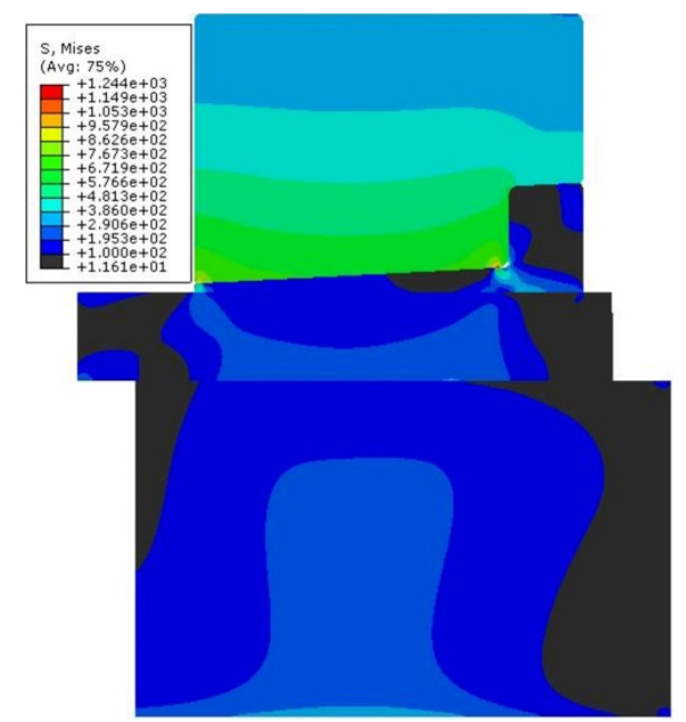

Figure 5. The distribution of Von Mises stress of the turbine shrink disk using the ideal model.

The maximum stress of the shaft, sleeve and outer ring lies in their internal surfaces, whereas the maximum stress $[18,19]$ of the inner ring lies in its external surfaces. Therefore, the axial nodes on the internal surfaces of the shaft, sleeve and outer ring, together with the nodes on the external surfaces of the inner ring, were selected to analyze the Von Mises stress of each component, which were used as the key parameters to determine whether the components produced plastic deformation or not. The contact pressure was analyzed by the CPRESS module of ABAQUS [20]. Figure 6 describes the distribution of contact pressure [21] on each mating surface under different assembly clearances. Figure 7 gives the distribution of the contact pressure on each mating surface [22] under different machining deviations $[23,24]$. Figure 8 shows the distribution of the contact pressure on each surface [25] under different propel travels.

\subsection{Assembly Clearance}

As shown in Figure 6, the variation trends of contact pressure for Models 1-3 with the axial-directional nodes are similar. For the same axial node of each component for the three models, there exist the following features: (1) comparing with Model 1, the contact pressures of Model 2 and 3 were reduced to different degrees; (2) Model 2 has less reducing trend, whereas Model 3 has more reducing trend; and (3) the assembly clearance has the greatest influence on contact pressure on the mating surfaces of the shaft and the sleeve.

Compared with ideal Model 1, the contact pressure recorded for Model 3 decreases by approximately $50 \mathrm{MPa}$ in Figure 6a. Therefore, the assembly clearance is positively proportional to the contact pressure on the mating surfaces. The maximum clearance has a great impact on the mating surfaces, whereas the minimum clearance has a minimal impact. 


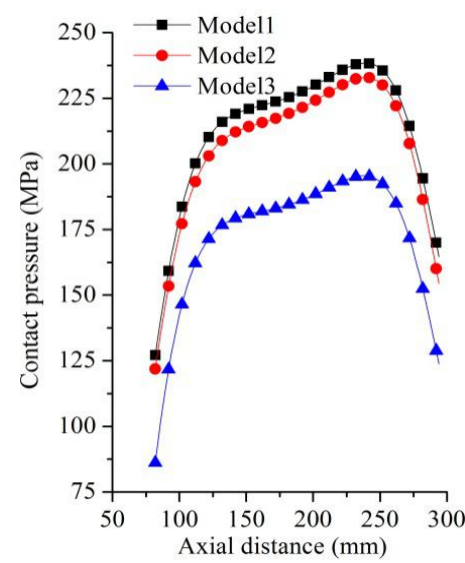

(a)

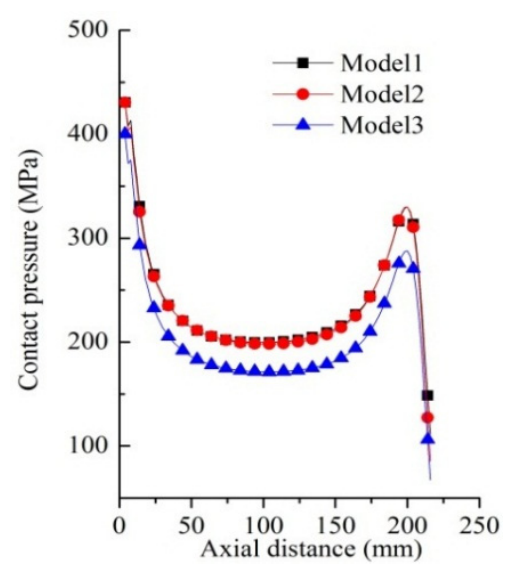

(b)

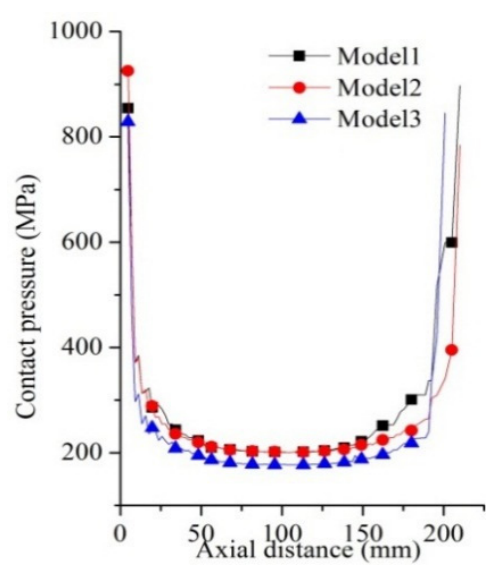

(c)

Figure 6. Distribution of contact pressure on each mating surface under different assembly clearances. (a) Mating surfaces of shaft and sleeve; (b) mating surfaces of sleeve and inner ring; (c) mating surfaces of inner ring and outer ring.

\subsection{Machining Deviations}

As shown in Figure 7, the variation trends of contact pressure on Model 1, 4 and 5 with the axial-directional nodes are similar. For the same axial node of each component, there exist the following features: the contact pressure of Model 5 has the largest value, model 1 has the second largest, and Model 4 has the smallest. The maximum difference of contact pressure is within $25 \mathrm{MPa}$ in Figure 7a. Thus, the interference magnitude is inversely proportional to the contact pressure on mating surfaces. Machining deviation within a reasonable range has a small impact on the contact pressure of mating surfaces.

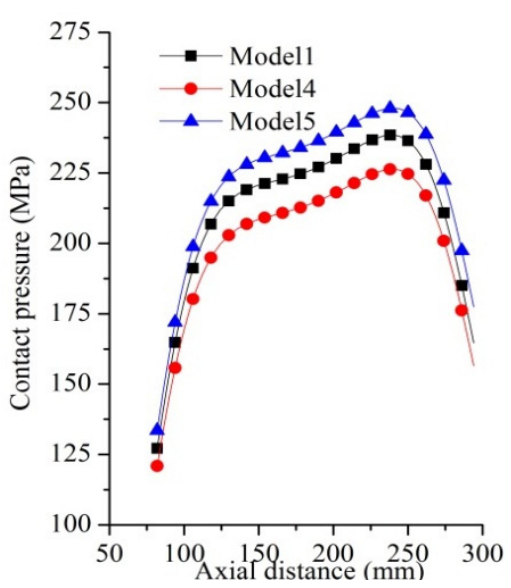

(a)

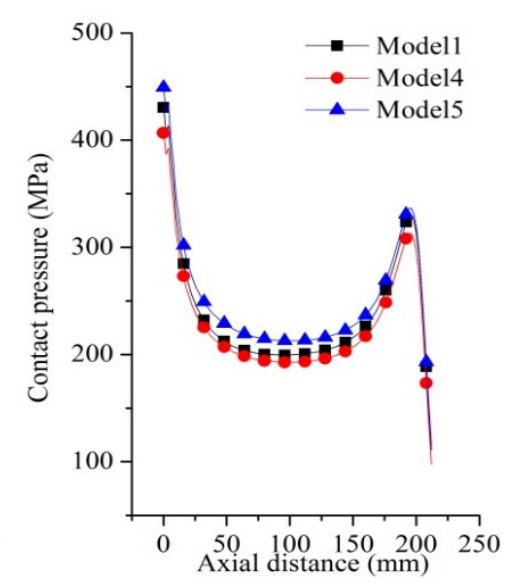

(b)

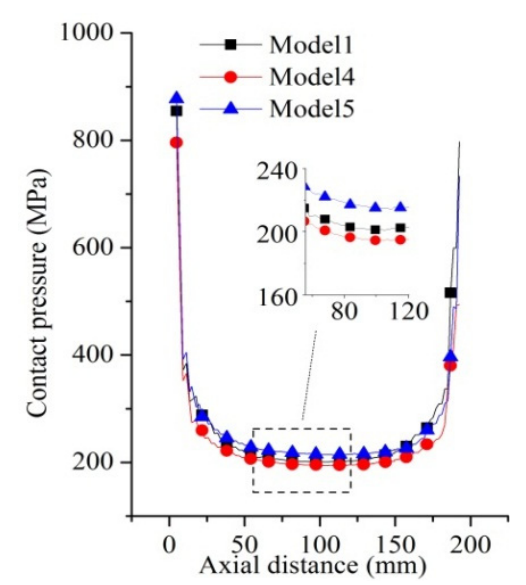

(c)

Figure 7. Distribution of the contact pressure on each mating surface under different machining deviations. (a) Mating surfaces of shaft and sleeve; (b) mating surfaces of sleeve and inner ring; (c) mating surfaces of inner ring and outer ring.

\subsection{Propel Travel}

From Figure 8, the distribution of the contact pressure of the mating surface between the shaft and the sleeve is low on both ends and high in the middle. Compared to the value of the same node, the contact pressure will increase with propel travel, and the increment is roughly a linearly proportional 
relationship. The contact pressure under full propel travel BD is approximately $50 \mathrm{MPa}$ higher than that under the minimum propel travel $\mathrm{BD}_{1}$.

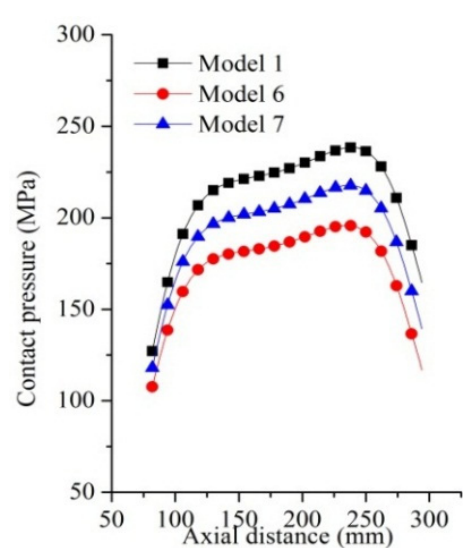

(a)

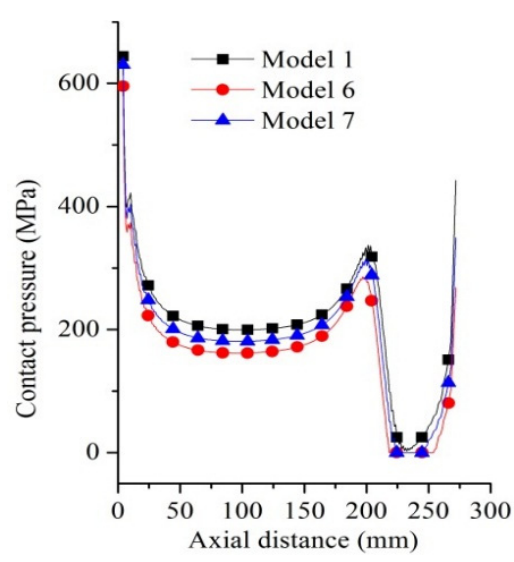

(b)

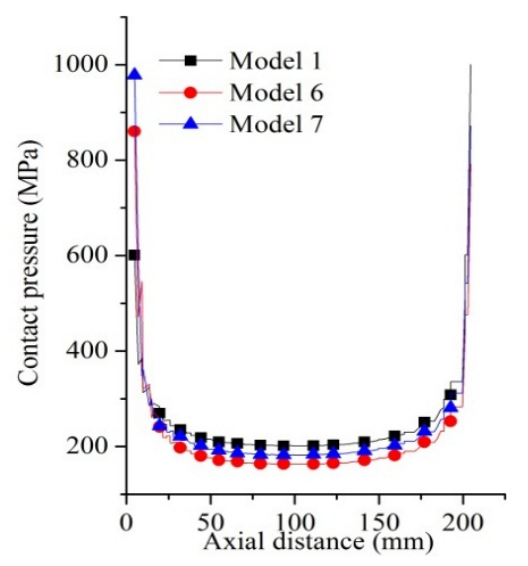

(c)

Figure 8. Distribution of the contact pressure on each mating surface under different propel strokes. (a) Mating surfaces of shaft and sleeve; (b) mating surfaces of sleeve and inner ring; (c) mating surfaces of inner ring and outer ring.

\subsection{Performance of the Models}

The load torque is the main parameter used to evaluate the load-carrying performance of a turbine shrink disk; the load torque relates to the following parameters: contact pressure, friction coefficient, length of mating surfaces and diameter of shaft. The corresponding load torque can be obtained from the contact pressure. The load-carrying performance of the turbine shrink disk can be directly judged by comparison to the related torque. A calculation equation of the loading torque can be deduced from Equation (1).

$$
M=\frac{\mu P \pi d^{2} l}{2}
$$

Using the curving integral of the mating surface between the shaft and the sleeve, the load torque of the turbine shrink disk can be obtained by combining it with Equation (13). The results of the load torque on each model and the relative error compared with model 1 (ideal model) are shown in Table 4 .

Table 4. Load torque of each model and relative error.

\begin{tabular}{|c|c|c|}
\hline Model Number & Torque $(\mathbf{k N} \cdot \mathbf{m})$ & Relative Error (\%) \\
\hline $1 \#$ & 3396 & 0.0 \\
\hline 2\# & 3245 & 4.4 \\
\hline 3\# & 2599 & 23.5 \\
\hline $4 \#$ & 3233 & 4.8 \\
\hline $5 \#$ & 3565 & 5.0 \\
\hline $6 \#$ & 2675 & 21.2 \\
\hline 7\# & 3033 & 10.7 \\
\hline
\end{tabular}

As shown in Table 4, compared with Model 1 (ideal model), the relative error of the load torque of Model 2 is $4.4 \%$, which shows that the minimum assembly clearance has little influence on the load torque and can meet the engineering design requirements. The relative errors of Model 4 and 5 are $4.8 \%$ and $5.0 \%$, respectively, which shows that the machining deviation range can also meet the engineering requirements. However, the relative error of Model 3 reaches 23.5\%, i.e., the maximum assembly clearance has a great effect on the load-carrying performance. Moreover, as the deviation of propel stroke between the design value and the practical value increases, the greater the weakening 
effect on load torque becomes. The full axial movement of the outer ring can guarantee the normal operation of the turbine shrink disk.

\section{Conclusions}

The key influencing factors on the load-carrying performance of a multiple-layer interference cylinder were studied by finite element simulation of a turbine shrink disk. The influences of assembly clearance, machining derivation, and propel stroke were obtained by analyzing the contact pressure of the mating surfaces and the load torque. The conclusions drawn are as follows.

(1) The theoretical model and finite element model of the turbine shrink disk were constructed, and a test device has been developed to verify the reasonability of the proposed models for load-carrying performance evaluation.

(2) Different assembly clearances have different effects on the load-carrying performance of a turbine shrink disk. The maximum assembly clearance has a large effect on the contact pressure of the mating surfaces and the load torque, whereas the effect of the minimum assembly clearance is relatively small. The effect of the assembly clearance must be taken into consideration during the design and calculation, and the assembly clearance should be reasonably determined.

(3) Machining deviation has a relatively small effect on the contact pressure and load torque of the mating surfaces. If the machining deviation is within reasonable range, then the load-carrying performance of a multiple-layer interference fit can be ensured.

(4) The propel stroke has a significant effect on the contact pressure of the mating surface and the load torque. To avoid the abnormal operation of equipment caused by insufficient load torque, the propel stroke during practical assembly should be at least 0.95 times that of the designed propel stroke.

Acknowledgments: This work was supported by the Shanxi Provincial Natural Science Foundation of China (No. 201601D011049) and the Shanxi Province special patent to promote implementation of the funds of China (No. 20161005), the Shanxi Provincial Key Research and Development Project (No. 201603D111017), and the National Science Foundation of China (No. U1610109 and 51505475).

Author Contributions: Jianmei Wang, Liang Tang and Ke Ning conceived and designed the experiments; Reza Malekian and Zhixiong Li performed the experiments; Ke Ning and Yinan Liang analyzed the data; Zhixiong Li and Yinan Liang contributed analysis tools; Jianmei Wang and Ke Ning wrote the paper.

Conflicts of Interest: The authors declare no conflict of interest.

\section{References}

1. Biron, G.; Vadean, A.; Tudose, L. Optimal design of interference fit assemblies subjected to fatigue loads. Struct. Multidiscip. Optim. 2013, 47, 441-451. [CrossRef]

2. Chakherlou, T.; Mirzajanzadeh, M.; Vogwell, J. Experimental and numerical investigations into the effect of an interference fit on the fatigue life of double shear lap joints. Eng. Fail. Anal. 2009, 16, 2066-2080. [CrossRef]

3. Truman, C.; Booker, J. Analysis of a shrink-fit failure on a gear hub/shaft assembly. Eng. Fail. Anal. 2007, 14, 557-572. [CrossRef]

4. Manuel, P.; Naoufel, N.; Marc, S. Study of an interference fit fastener assembly by finite element modeling, analysis and experiment. Int. J. Interact Des. Manuf. 2012, 6, 171-177.

5. Boutoutaou, H.; Bouaziz, M.; Fontaine, J. Modeling of interference fits taking form defects of the surfaces in contact into account. Mater. Design 2011, 32, 3692-3701. [CrossRef]

6. Mack, W.; Plöchl, M. Transient heating of a rotating elastic-plastic shrink fit. Int. J. Eng. Sci. 2000, 38, 921-938. [CrossRef]

7. Jahed, H.; Farshi, B.; Karimi, M. Optimum autofrettage and shrink-fit combination in multi-layer cylinders. J. Pressure Vessel Tech. 2006, 128, 196-200. [CrossRef]

8. Sogalad, I.; Ashokaa, H. Influence of cylindricity and surface modification on load bearing ability of interference fitted assemblies. Precision Eng. 2012, 36, 629-640. [CrossRef] 
9. Cheng, D.X. Handbook of Mechanical Design, Connection and Fastening; Chemical Industry Press: Beijing, China, 2004; Volume 2, pp. 276-290.

10. Wang, J.; Tang, L. Shrink Disk Design Theory and Methodology; Metallurgical Industry Press: Beijing China, 2014; Volume 2, p. 41.

11. Zhang, H.; Liao, A.; Wu, C. Numerical simulation of contact problems in vane machinery by a parametric quadratic programming method. Arch. Appl. Mech. 2007, 77, 421-437. [CrossRef]

12. Wang, J.; Tao, D.; Huang, Q. Algorithm research on contact pressure and magnitude of interference for multi-layer cylinder's interference fit. Eng. Mech. 2013, 30, 270-275.

13. Özel, A.; Temiz, S.; Aydin, M.; Şen, S. Stress analysis of shrink-fitted joints for various fit forms via finite element method. Mater. Design 2005, 26, 281-289. [CrossRef]

14. Sun, M.; Lu, S.; Li, D.; Li, Y.; Lang, X.; Wang, S. Three-dimensional finite element method simulation and optimization of shrink fitting process for a large marine crankshaft. Mater. Design 2010, 31, 4155-4164. [CrossRef]

15. Zeng, F.; Chen, G.; Zhou, Z. Fretting analysis of interference fitting of wheel-set based on ANSYS. J. Mech. Eng. 2011, 47, 121-125. [CrossRef]

16. Pedersen, P. On shrink fit analysis and design. Comput. Mech. 2006, 37, 121-130. [CrossRef]

17. Wang, J.; Kang, J.; Tang, L. Theoretical and experimental studies for wind turbine's shrink disk. Proc. Inst. Mech. Eng. C J. Mech. Eng. Sci. 2015, 229, 325-334.

18. Zhang, X.; Shao, J.; An, W.; Yang, T.; Malekian, R. An improved time-frequency representation based on nonlinear mode decomposition and adaptive optimal kernel. Elektron. Elektrotech. 2016, 22, 52-57.

19. Jin, X.; Shao, J.; Zhang, X.; An, W.; Malekian, R. Modeling of nonlinear system based on deep learning framework. Nonlinear Dy. 2016, 84, 1327-1340. [CrossRef]

20. Wang, Z.; Ye, N.; Malekian, R.; Wang, R.; Li, P. TMicroscope: Behavior perception based on the slightest RFID tag motion. Elektron. Elektrotech. 2016, 22, 114-122. [CrossRef]

21. Malekian, R.; Bogatinoska, D.C.; Karadimce, A.; Trengoska, J.; Nyako, W.A. A novel smart ECO model for energy consumption optimization. Elektron. Elektrotech. 2015, 21, 75-80.

22. Prabu, K.; Kumar, D.S.; Malekian, R. BER Analysis of BPSK-SIM-based SISO and MIMO FSO systems in strong turbulence with pointing errors. Opt. Inter. J. Light Electron. Opt. 2014, 125, 6413-6417. [CrossRef]

23. Modieginyane, K.M.; Letswamotse, B.B. Software defined wireless sensor networks (SDWSNs) application opportunities for efficient network management: A survey. Comput. Electr. Eng. J.. (in press).

24. Wang, Z.; Ye, N.; Xiao, F.; Wang, R. TrackT: Accurate tracking of RFID tags with mm-level accuracy using first-order taylor series approximation. AD Hoc Netw. 2016, 53, 132-144. [CrossRef]

25. Shao, J.; Wang, L.; Zhao, W.S.; Zhong, Y.Q. An improved synchronous control strategy based on fuzzy controller for PMSM. Elektron. Elektrotech. 2014, 20, 17-23.

(C) 2017 by the authors. Licensee MDPI, Basel, Switzerland. This article is an open access article distributed under the terms and conditions of the Creative Commons Attribution (CC BY) license (http:/ / creativecommons.org/licenses/by/4.0/). 